\title{
Current vortices and magnetic fields driven by moving polar twin boundaries in ferroelastic materials
}

\author{
Guangming $\mathrm{Lu}^{1,2}$, Suzhi Li $\mathbb{D}^{1 凶}$, Xiangdong Ding $\mathbb{D}^{1 凶}$, Jun Sun ${ }^{1}$ and Ekhard K. H. Salje $\mathbb{D}^{1,2 \bowtie ~}$
}

Ferroelastic twin boundaries often have properties that do not exist in bulk, such as superconductivity, polarity etc. Designing and optimizing domain walls can hence functionalize ferroelastic materials. Using atomistic simulations, we report that moving domain walls have magnetic properties even when there is no magnetic element in the material. The origin of a robust magnetic signal lies in polar vortex structures induced by moving domain walls, e.g., near the tips of needle domains and near domain wall kinks. These vortices generate displacement currents, which are the origin of magnetic moments perpendicular to the vortex plane. This phenomenon is universal for ionic crystals and holds for all ferroelastic domain boundaries containing dipolar moments. The magnetic moment depends on the speed of the domain boundary, which can reach the speed of sound under strong mechanical forcing. We estimate that the magnetic moment can reach several tens of Bohr magnetons for a collective thin film of 1000 lattice planes and movements of the vortex by the speed of sound. The predicted magnetic fields in thin slabs are much larger than those observed experimentally in $\mathrm{SrTiO}_{3} / \mathrm{LaAlO}_{3}$ heterostructures, which may be due to weak (accidental) forcing and slow changes of the domain patterns during their experiments. The dynamical multiferroic properties of ferroelastic domain walls may have the potential to be used to construct localized magnetic memory devices in future.

npj Computational Materials (2020)6:145; https://doi.org/10.1038/s41524-020-00412-5

\section{INTRODUCTION}

Interface physics has attracted much interest in the field of ferroelastic materials. Known as domain boundary engineering, ferroelastic materials can be functionalized by designing and optimizing domain walls ${ }^{1,2}$. Many studies have observed that interface possess properties that do not exist in bulk, such as the superconductivity $^{3-5}$, ferroelectricity ${ }^{6,7}$. For example, twin walls are known to be superconductive in $\mathrm{WO}_{3-\mathrm{x}}{ }^{8,9}$. Another common effect is that polarity is generated near domain walls via the flexoelectric effect ${ }^{10,11}$, e.g., in $\mathrm{CaTiO}_{3}{ }^{12-14}$. Besides the isolated domain walls, emerging functional properties have also been found near the interfaces between different oxides ${ }^{15-17}$ or different orientations of the same oxide ${ }^{18}$. For instance, the two-dimensional electron gas at the interface between $\mathrm{SrTiO}_{3} / \mathrm{LaAlO}_{3}$ based heterostructures has been verified to be responsible for the occurrence of the interfacial conductivity ${ }^{19-21}$ and superconductivity ${ }^{22,23}$. Among these functional properties, magnetism has rarely been reported to emerge from twin walls. Although some ideas have been proposed to explore the possible magnetisms inside ferroelastic heterostructures ${ }^{24,25}$, the corresponding magnetic signals were too weak for practical applications or even to be measured experimentally. Recently, within the framework of dynamical multiferroicity ${ }^{26,27}$, magnetic signals were shown to emerge from inside moving ferroelectric domain walls. In a similar approach, we expand this idea to the moving kinks in twin walls and needle tips in ferroelastic materials. We argue, using atomistic simulations, that topological nano-structures (stripe twins or needles domains ${ }^{28-36}$ etc.) can, thus, generate noticeable magnetism. This behavior relies on the nucleation and movement of kinks in twin walls $^{11,37-39}$. These kinks were shown before to be highly mobile and can be accelerated by external strain. They form polar vortices near the kinks, which, in turn, are the origin of displacement currents and hence of magnetism.
Using atomistic simulations, we report a robust magnetic signal induced by moving ferroelastic domain walls based on a twodimensional theoretical model (the sample is charge neutral, no free electrons exist) ${ }^{11}$. The displacement current and magnetic moment are comparable with mobile polar structures and the formation of polar vortices proposed in refs ${ }^{26,27}$, where magnetic moments amounted to $\sim 10^{-6} \mu_{B}$ in the domain walls. Here we describe much stronger magnetism by moving ferroelastic needle domains although the magnetic moment density is smaller than that in ref. ${ }^{26}$. The primary magnetic field is not generated at the twin walls, but near twin walls, where the displacement current vortices are induced by kinks in the domain walls. Our findings are potentially important for designing nano-devices based on the magnetic field produced by the moving polar ferroelastic structures in thin slabs instead of interfaces in heterostructures.

\section{RESULTS}

Displacement current and magnetic field driven by a moving single kink

First we analysed toy models based on Landau springs to describe the interatomic potential in ferroelastic materials (see "Methods" section). The ferroelastic shear angles at the equilibrium state are chosen to be $2^{\circ}$ and $4^{\circ}$, corresponding to materials like $\mathrm{SrTiO}_{3}$ and $\mathrm{LaAlO}_{3}$ (see "Methods" section). In this paper, we show the results using the model with shear angle of $2^{\circ}$ (see the simulation results for $4^{\circ}$ model in Supplementary materials). In order to illustrate the basic mechanism how a magnetic moment is generated, we initially focus on a single kink motion inside a ferroelastic twin wall under free boundary conditions (Supplementary Fig. S1). When the system relaxes, the kink moves from left to right. The moving kink induces relative displacements of nearby cations and anions at a time interval $\Delta t$, as shown in Fig. 1a. The relative

${ }^{1}$ State Key Laboratory for Mechanical Behavior of Materials, Xi'an Jiaotong University, Xi'an 710049, China. ${ }^{2}$ Department of Earth Sciences, University of Cambridge, Cambridge

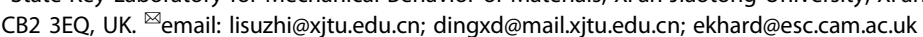



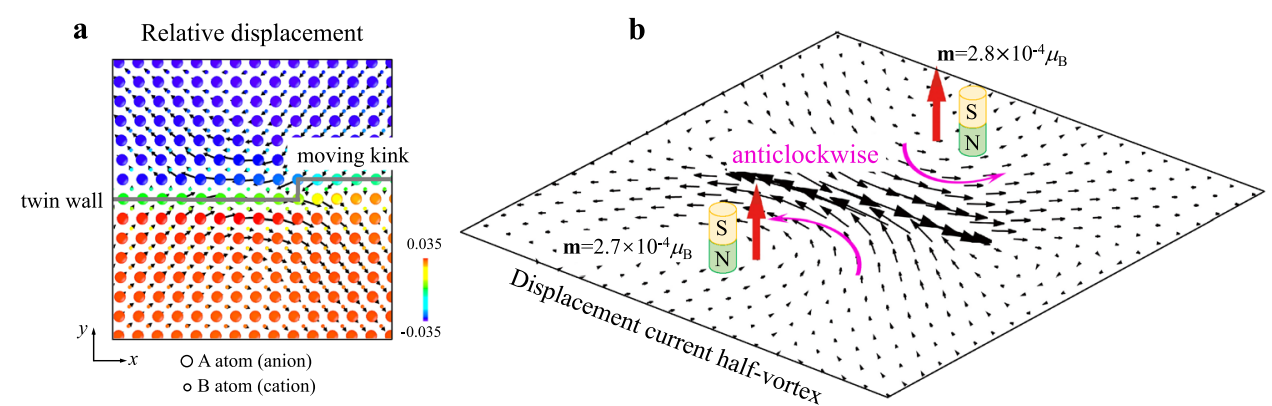

Fig. 1 Displacement currents and the corresponding magnetic fields produced by moving a kink inside a ferroelastic twin wall. a Relative displacements of cations and anions near the moving kink during a time interval of $0.5 \mathrm{ps}$. The colors are coded by the atomic-level shear strain. Ionic displacements are amplified by a factor of 50 for clarity. b Two half-vortices of the displacement currents rotate in the same direction in both domains. The current density was calculated based on the relative displacements of anions and cations (see "Methods" section). The local displacement current is $\sim 10^{-19}$ A. The magnetic field is $\sim 2.7 \times 10^{-4} \mu_{B}$ for each half-vortex (see "Methods" section). The current vectors are amplified by a factor of $2 \times 10^{17}$ for clarity.

displacements of the cations and anions on upper and lower part of the domain are symmetrical. The moving kink generates displacement currents (see "Methods" section and Supplementary Fig. S2), where the displacement currents (per time interval) form vortices on either side of the twin wall. The important observation is that these vortices have the same sense of rotation (Fig. 1b). As the two displacement currents rotate in the same direction, their induced magnetic moments are additive. Using the "Biot-Savart" law, the magnetic moment is calculated to be $\sim 2.7 \times 10^{-4} \mu_{B}$ (see "Methods" section and Supplementary Fig. S3), where $\mu_{B}=$ $9.274 \times 10^{-24} \mathrm{JT}^{-1}$ is the magnetic moment of an electron. This fact is the key point of our argument. It is different from the idea in refs 26,27 , where the displacement current is considered inside domain walls. In our approach we consider displacement currents outside domain walls where displacement currents are induced by kinks and other topological defects. Our walls are ferroelastic and need not to be ferroelectric. This opens applications to many more materials outside ferroelectrics.

\section{Displacement current and magnetic field generated by moving} needle domains

We now concentrate on topological structures where kinks are predominant. One such configuration is a needle domain where kinks occur inside the needle tip-but not in the shaft ${ }^{28,29,37,40-44}$. The simulation box has the size $5.6 \mathrm{~nm}$ in $x$ direction and $15.0 \mathrm{~nm}$ in $y$ direction. We first explore the behavior of a single needle domain. Moving needle domains are extremely common in ferroelastic materials. Their dynamics have been described for several decades ${ }^{28,29,37,40-45}$. The needle domains contain kinks near the tip ${ }^{46}$, where the domain walls appear mesoscopically bent. We first consider three kink pairs inside the domain structure (Supplementary Fig. S4). The upper and lower sample surfaces were fixed. The local strain distributions near the middle kink pair exhibit typical Eshelby strain patterns ${ }^{46}$ (Supplementary Fig. S4). Electric dipoles occur inside twin walls (Supplementary Fig. S5) due to the flexoelectric effect in the twin walls and at the location of large local inhomogeneous strains (Supplementary Fig. S4). The out-of-twin-plane dipoles are activated by the flexoelectric effect of the strain gradients (Supplementary Fig. S5). When needle domains advance and retreat, they carry these dipoles with them and generate dipolar fields that change coherently. The needle movement, and hence the kinks and dipolar fields, oscillate under oscillatory forcing.

We then applied a simple shear to drive the movement of the needles and the kinks in the domain boundaries with a strain rate of $2.7 \times 10^{8} \mathrm{~s}^{-1}$ (Supplementary Fig. S4). The kinks have the same orientation and produce repulsive force between them. Under weak stress, kink pair 1 is firstly activated to move, followed by the other two kink pairs. Figure $2 a$ shows the atomic configuration of the moving kink pair 3. The relative displacement of cations and anions are generated around the kink pair, as shown in Fig. 2b. Figure $2 \mathrm{c}$ shows the corresponding displacement current and the magnetic moment perpendicular to the atomic plane. The displacement current is of the order of $\sim 10^{-18} \mathrm{~A}$. The current density map for the kink pair is composed of one displacement current (current vortex) inside the needle and two half-displacement currents on each side of the kink (Fig. 2c). The magnetic moment corresponding to the middle full current vortex is $1.2 \times 10^{-3} \mu_{B}$. We roughly estimated the magnetic moment density and found a value of $60 \%$ of the moving ferroelectric twin walls $^{26}$ (see "Methods" section). The magnetic moment depends on the distance between kinks and the needle thicknesses (Supplementary Fig. S6). The increase of the magnetic moment with increasing kink-kink distance is attributed to the increase of the displacement current while the flexoelectric effect between kink pairs decreases so that the simulated magnetic moment is a compromise between these two factors. In our simulations, kink pairs reach a constant speed of $0.72 \mathrm{~km} \mathrm{~s}^{-1}$ (pair 1), $0.80 \mathrm{~km} \mathrm{~s}^{-1}$ (pair 2), and $1.03 \mathrm{~km} \mathrm{~s}^{-1}$ (pair 3), as shown in Fig. 2d. The small differences manifest that moving needle domains slightly change their shape under high speeds, known as "sharpening the needle". Needles slow down when approaching the lower fixed surface. Two snapshots ( $\Delta t=0.5 \mathrm{ps}$ ) of the moving trajectory of every kink pair were then chosen to calculate the current density near every kink pair ${ }^{26,27,47}$. In comparison with the $2^{\circ}$ model, the $4^{\circ}$ degree model produces stronger displacement currents and stronger magnetic fields (Supplementary Fig. S6). The ferroelastic switching is achieved by moving kinks in the $4^{\circ}$ model, which induces a larger relative displacement of cations and anions.

Another typical microstructure in ferroelastic materials is the comb pattern $28,29,37,40-45,48$, which consists of needle arrays with various distances between the needles. In our model, the stressdriven propagation of comb pattern consists of three vertical thin needles with 4 atomic layers for each (Supplementary Fig. S7). By controlling the external stress, vertical needles are stabilized at the same distance from the surface with equal needle distances of $1.5 \mathrm{~nm}$. The local strain fields show typical Eshelby patterns (Supplementary Fig. S7), similar to that in the kink pair system. The profile of each needle consists of two parts: the needle tip and double kinks inside the walls near the tip. The planar dipole configurations now show antiparallel dipole configurations with out-of-twin-plane dipoles (Supplementary Fig. S8).

A shear deformation drives the propagation of the comb pattern with a strain rate $2.7 \times 10^{8} \mathrm{~s}^{-1}$. All needle tips are energetically equivalent. The activation barriers for driving needle tips movement are therefore the same. The tips are activated at the same time with a gradual increase of speed before reaching a steady state at a velocity of $0.24 \mathrm{~km} \mathrm{~s}^{-1}$. They finally slow down 


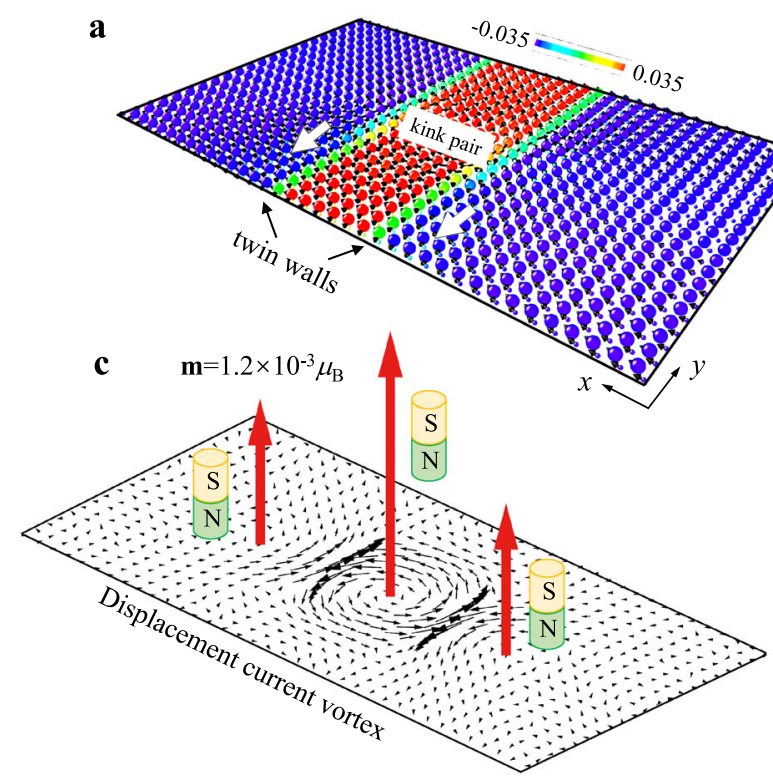

b

Relative displacement

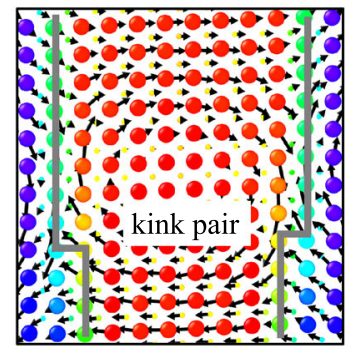

d

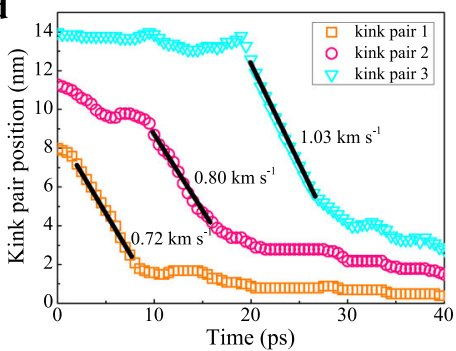

Fig. 2 Displacement currents and the corresponding magnetic fields generated by a moving kink pair. a The atomic configuration of the movement of kink pairs driven by external stress. The colors are coded by the atomic-level shear strain. $\mathbf{b}$ The relative displacements of cations and anions near the kink pairs within $0.5 \mathrm{ps}$. Ionic displacements are amplified by a factor of 50 for clarity. c One displacement current vortex is inside the vertical needle and two displacement current half-vortices outside the needle. The local displacement current is $\sim 10^{-18} \mathrm{~A}$. The generated magnetic moment is $1.2 \times 10^{-3} \mu_{B}$. The current densities in the vector maps are amplified by a factor of $2 \times 10^{17}$ for clarity. $\mathbf{d}$ Time evolution of positions of three kink pairs. The velocity was estimated when the kinks move steadily.

when moving close to the lower surface. Two atomic configurations with a time interval of $\Delta t=3.0 \mathrm{ps}$ during the steady-state motion were chosen to calculate the local displacement current density and the corresponding magnetic moment (Supplementary Fig. S9). Displacement currents are observed between the vertical needles, as shown in Supplementary Fig. S9. The strength of the local displacement current density is weaker than that in the kink pair system, with magnetic moments of the same magnitude when the geometry effect of the displacement current (larger vortex diameters) are taken into account. The weaker displacement current can be explained by the slower speed of the needle tips and a smaller flexoelectric effect for needle tips with larger tip-tip distances. By comparing the magnetic moment in the $2^{\circ}$ model and the $4^{\circ}$ model (Supplementary Fig. S9), we confirm that the larger flexoelectric effect increases the resulting magnetic field.

To generate stronger magnetic fields, we shortened the intertip distances to $0.7 \mathrm{~nm}$ (Fig. 3a). The comb pattern now consists of 5 vertical needle domains with a thickness of the needle of only 3 atomic layers. The symmetry breaking of every single needle is enhanced by a strong strain interaction between nearby vertical needles. The application of shear strain moves the comb pattern downwards. Two snapshots on the moving trajectory were used to calculate the displacement current and magnetic moment. The local displacement current increases relative to the sparse pattern because of stronger strain interactions between vertical needles (Fig. 3b). Simultaneously, the diameter of each single current vortex becomes smaller compared with the previous model. The calculated magnetic moment is $7.3 \times 10^{-4} \mu_{B}$ for each displacement current vortex (Fig. 3c), which is smaller than the value of $1.1 \times 10^{-3} \mu_{B}$ for individual needles in Supplementary Fig. S9. However, the total magnetic moment of the entire comb pattern becomes larger due to a higher density of needle domains (Fig. 3c). The speed of the comb pattern in Fig. $3 \mathrm{~d}$ is $0.24 \mathrm{~km} \mathrm{~s}^{-1}$. The system with larger shear angle of $4^{\circ}$ generates an enhanced displacement current and magnetic moment (Supplementary Fig. S10).

\section{DISCUSSION}

The displacement current and the corresponding magnetic field depend on the distances between kink pairs or the needle density inside the comb patterns. Such tunability stems from the generation of different dipolar moments between needle tips that vary the diameter of the displacement current vortices. The generated magnetic field does not only depend on the moving speed of the polar twin boundaries, but also on the flexoelectricity and geometric configurations of the twin structures. The flexoelectricity characterizes the coupling between strain gradients and polarization. Structural defects carrying strong strain gradients, like moving kinks, needles, and junctions, induce large displacement currents and hence a large magnetic moment. For example, enhancing the flexoelectric polarization by increasing the ferroelastic shear angle produces stronger magnetic fields. Magnetic fields produced by the fast movement of our ferroelastic twins are much larger than the recent investigations on the heterogeneous structures near $\mathrm{LaAlO}_{3}$ and $\mathrm{SrTiO}_{3}$ (LAO/STO) interfaces ${ }^{24,25}$. In these observations, the occurrences of the magnetic signals were explained in terms of the couplings between lattices and spins or free electrons near the LAO/STO interfaces or the domain walls below $\mathrm{T}_{-} \mathrm{c}=105 \mathrm{~K}$ for $\mathrm{SrTiO}_{3}$. Such couplings are normally very weak in ferroelastic materials, and the observed magnetic signals are therefore very small. The strong increase of the magnetic field in our simulations stems from the fast movement of kinks in ferroelastic walls, which produces significant displacement currents and hence potentially bigger magnetic signals than those observed in the LAO/STO interface.

Ferroelastic thin slabs often consist of more than 1000 atomic layers. In addition, the movement of the polar ferroelastic twin walls is often much faster than those shown in our simulations (even supersonic speed has been found ${ }^{46}$ ). In a rough estimation we use the speed of sound of aluminium $\left(6.42 \mathrm{~km} \mathrm{~s}^{-1}\right)$ as the speed of the patterns and extend the slab thickness to 1000 layers. Under these circumstances we find a very large magnetic moment $\left(\mathbf{m}=78.11 \mu_{B}\right)$ during the motion of the ferroelastic comb structures (Fig. 3). Such large magnetic moments are sufficient for applications as magnetic memory devices. Nevertheless, the 

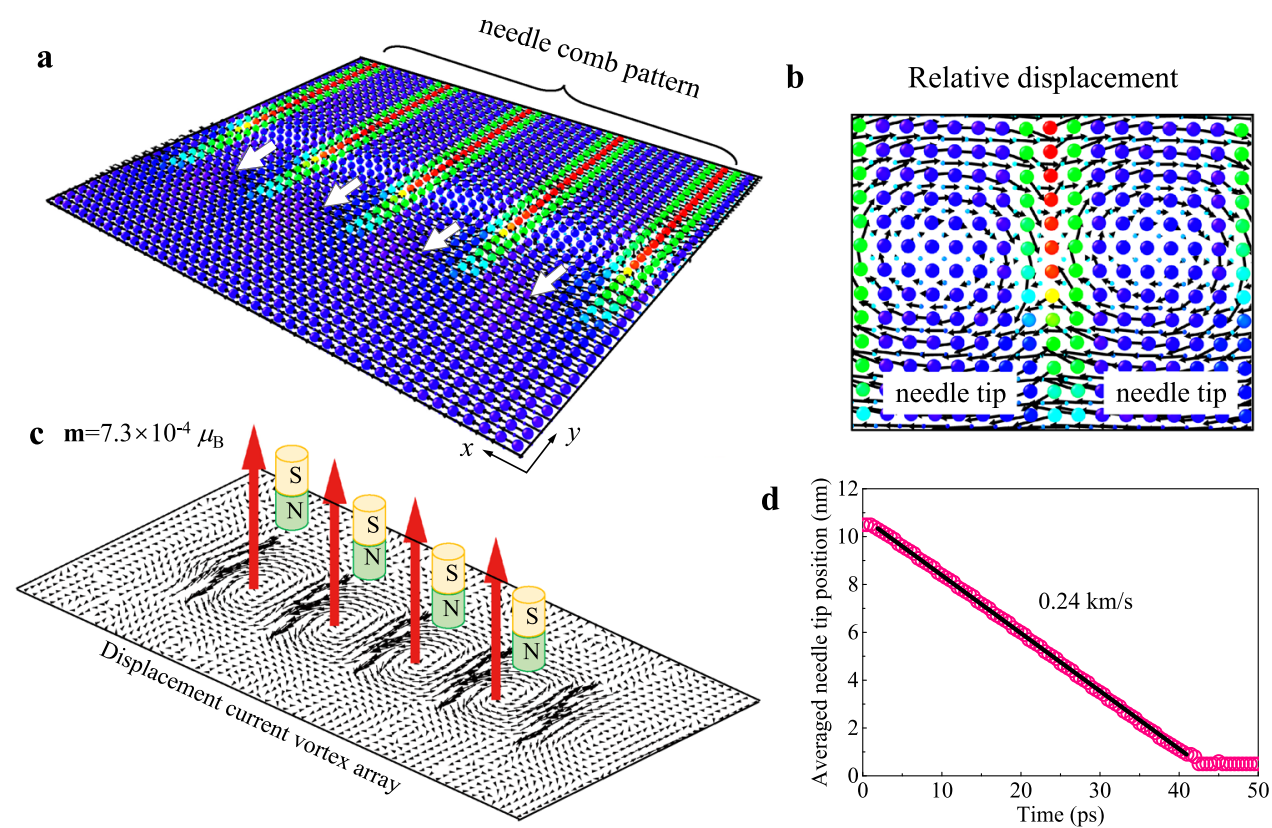

Fig. 3 Displacement currents and the corresponding magnetic fields produced by the propagation of the comb pattern. a The atomic images of movement of needle domains by the external stress. The distance between parallel needles is $0.7 \mathrm{~nm}$. The colors are coded according to the atomic-level shear strain. $\mathbf{b}$ The relative displacement of cations and anions around the kink pairs within 3.0 ps. lonic displacements are amplified by a factor of 30 for clarity. The symmetric structure of every single domain is broken by strong interactions between dense needle domains. c The displacement currents near the moving needle tips (displacement current vortices) generate magnetic fields in the out-of-twin-plane direction. The current density was calculated using the relative displacements of anions and cations within a time interval of $3.0 \mathrm{ps}$. The current densities in the vector maps are amplified by a factor of $1 \times 10^{18}$ for clarity. $\mathbf{d}$ The averaged position of the needle tips with five needles as a function of time during needle propagation.

moment density of $3.9 \times 10^{-5} \mu_{B} \mathrm{fu}^{-1}$ is very small compared with magnetic materials with typical moment densities per formula unit (fu) of $2 \mu_{B} \mathrm{fu}^{-1}$.

We finally proposed an experimental arrangement to measure the magnetic field. Recently developed experimental technique could prepare ferroelastic materials with a high density of domain walls. With such a sample, the first step is to apply a local stress in an atomic force microscope (AFM) to move atomic kinks and needle domains inside the polar twin walls, as shown in Fig. $4 a$, b. Simultaneously the magnetic field is measured using a superconducting quantum interference device (SQUID) ${ }^{24,25}$. This experimental arrangement is similar to that proposed to measure the magnetization in a moving ferroelectric domain wall ${ }^{26}$, while has a major difference. In order to measure the magnetic signal generated by the ferroelectric walls, a pick-up loop is positioned on top of the ferroelectric wall. Such high-resolution SQUID pickup loops have typically one micro-meter diameter and are expected to be miniaturized further in future. The magnetic fields emerge from the displacement current vortices near the moving twin walls where the diameter of the current loop can be approximated by the dimension of the pick-up coil. A measurable magnetic signal is then expected to be observable directly in SQUID experiments.

In ferroelectric/ferroelastic material, such as $\mathrm{BaTiO}_{3}$, the required thin stripes with periodic twins (similar to Fig. 3, but with larger twin spaces) have been successfully fabricated and carefully controlled ${ }^{49}$. Other materials like $\mathrm{LaAlO}_{3}$ and $\mathrm{Pb}_{3}\left(\mathrm{PO}_{4}\right)_{2}$ were equally used to control the ferroelastic domain patterns ${ }^{50,51}$. The design of nano-devices based on the magnetic field produced by the moving polar ferroelastic twin walls then might be possible based on our proposed coupling mechanism between ferroelastic strain, polarity, and magnetic fields.

\section{METHODS}

Interatomic potential and model construction

Our simulations are based on a two-dimensional ionic spring model ${ }^{11}$ with two base atoms $(A$ and $B$ ), carrying negative and positive charges, respectively. The needle domain structures ( $A$ sublattice) were constructed by using nonlinear Landau springs while the interactions between $B$ atoms and $A-B$ atoms are purely harmonic to exclude any additional polar instabilities in bulk. We constructed two models with shear angles of $2^{\circ}$ and $4^{\circ}$, respectively. The construction of the $2^{\circ}$ model were inspired by $\mathrm{SrTiO}_{3}$ with T_c $=105 \mathrm{~K}$. The construction of the $4^{\circ}$ model is inspired by the fact that most typical ferroelastic shear angels are below $4^{\circ}$, such as $\mathrm{LaAlO}_{3}{ }^{43}$, $\mathrm{CaTiO}_{3}{ }^{12-14}$. The $4^{\circ}$ model can be used to explore the possible maximum value of magnetic signal we may detect. All parameters for the two models are shown in Table $I$ in the Supplementary Materials. The polarity occurs along the twin walls due to strong flexoelectricity. Additional long-range Coulomb forces were considered between $A-A, A-B$, and $B-B$ atoms. Free boundary conditions were used in both $x$ and $y$ directions. Both directions are charge free (no excess charges) by changing charges of the surfaces layers to be one half of that in the inner layers ${ }^{33}$. The temperature of the sample was controlled by the Nosé-Hoover thermostat ${ }^{52,53}$. All the calculations were carried out with the LAMMPS code $^{54}$.

\section{Calculation of displacement current density}

The displacement current density in each unit cell is calculated as $\mathbf{j}=\mathbf{j}_{\text {cation }}+\mathbf{j}_{\text {anion }}=Q^{+} \mathbf{v}_{\text {cation }}+Q^{-} \mathbf{v}_{\text {anion }}=Q^{+} \frac{\mathbf{s}_{\text {cation }}}{\Delta t}+Q^{-} \frac{\boldsymbol{s}_{\text {anion }}}{\Delta t}$ (ref. ${ }^{47}$ ), where $\mathbf{j}_{\text {cation }}$ and $\mathbf{j}_{\text {anion }}$ are the current density contributed by cation and anion, $Q^{+}$and $Q^{-}$are the amount of charge carried by cation and anion, $\mathbf{v}_{\text {cation }}$ and $\mathbf{v}_{\text {anion }}$ are the moving velocity of cation and anion, $\mathbf{s}_{\text {cation }}$ and $\boldsymbol{s}_{\text {anion }}$ are the moving distances of cation and anion during a time interval $\Delta t$. More details are shown in Supplementary Fig. S2 and refs $26,27,47$.

Calculation of magnetic moment produced by the displacement current

The magnetic moment perpendicular to the current vortex plane can be calculated as $\mathbf{m}=\frac{1}{2} \sum_{i} \frac{Q_{i}}{2 M_{i}} \mathbf{L}_{i}\left(\right.$ ref. ${ }^{47}$ ), where $Q_{i}$ is the amount of charge, $M_{i}$ is 


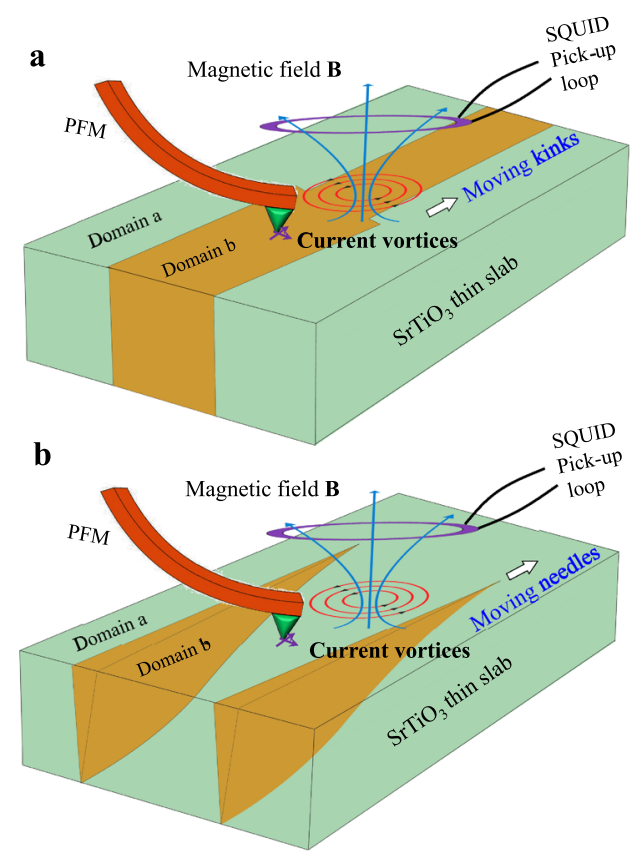

Fig. 4 Experimental arrangements for measuring displacement currents and the corresponding out-of-twin-plane magnetic field. The signals are generated by (a) moving kinks and (b) moving needles in a ferroelastic material. The local stress is applied by the AFM tip to drive the motion of the polar ferroelastic twin walls.

the atomic mass and $\mathbf{L}_{i}$ is the orbital angular momentum for the $i$ th moving particle. The direction of $\mathbf{L}_{i}$ is proportional to the vector product $\mathbf{r}_{i} \times \mathbf{v}_{i}$. The magnetic moment can be further calculated as $\mathbf{m}=\frac{1}{2} \sum_{i} Q_{i}\left(\boldsymbol{r}_{i} \times \boldsymbol{v}_{i}\right)=\frac{1}{2} \sum_{i} Q_{i}\left|\mathbf{r}_{i}\right|\left|\mathbf{v}_{i}\right| \sin \theta_{i}$, where $\mathbf{r}_{i}$ is the position vector, $\mathbf{v}_{i}$ is the moving velocity and $\theta_{i}$ the angle between the position vector and moving direction for the ith particle. More details are shown in Supplementary Fig. S3 and refs $26,27,47$.

\section{Estimation of magnetic moment density}

We chose a kink pair to estimate the magnetic moment density (Fig. 2). For a moving kink pair with velocity $1.03 \mathrm{~km} \mathrm{~s}^{-1}$, the induced magnetic moment is $\mathbf{m}=1.2 \times 10^{-3} \mu_{B}$. The area of displacement current vortex contains ca. 81 unit cells. The relevant volume $V$ is then one atomic layer times 81 unit cells and the moment density can be approximated as $\mathbf{m} / V=1.2 \times 10^{-3} \mu_{B} /(1$ atomic layer $\times 81$ unit cells $)$. Comparing with the results in ref. ${ }^{26}$, their magnetic moment is $\mathbf{m}=2.47 \times 10^{-5} \mu_{B}$ per unit cell right at the moving ferroelectric domain wall where the volume is one unit cell, i.e., $\mathbf{m} / V=2.47 \times 10^{-5} \mu_{B} /(1$ unit cell). The ratio between the kink model can then be roughly estimated to be $60 \%$ of the domain walls ${ }^{26}$.

\section{DATA AVAILABILITY}

The data that support the findings within this paper are available from the corresponding authors upon reasonable request.

\section{CODE AVAILABILITY}

The related codes are available from the corresponding authors upon reasonable request.

Received: 11 March 2020; Accepted: 2 September 2020; Published online: 25 September 2020

\section{REFERENCES}

1. Salje, E. K. H. Multiferroic domain boundaries as active memory devices: trajectories towards domain boundary engineering. ChemPhysChem 11, 940-950 (2010).
2. Salje, E. K. H. Domain boundary engineering-recent progress and many open questions. Phase Transit. 86, 2-14 (2013).

3. Yang, Z., Lange, M., Volodin, A., Szymczak, R. \& Moshchalkov, V. V. Domain-wall superconductivity in superconductor-ferromagnet hybrids. Nat. Mater. 3, 793-798 (2004)

4. Houzet, M. \& Buzdin, A. I. Theory of domain-wall superconductivity in superconductor/ferromagnet bilayers. Phys. Rev. B 74, 214507 (2006).

5. Catalan, G., Seidel, J., Ramesh, R. \& Scott, J. F. Domain wall nanoelectronics. Rev. Mod. Phys. 84, 119-156 (2012).

6. Salje, E. K. H., Li, S., Zhao, Z., Gumbsch, P. \& Ding, X. Polar twin boundaries and nonconventional ferroelectric switching. Appl. Phys. Lett. 106, 212907 (2015).

7. Lu, G., Li, S., Ding, X., Sun, J. \& Salje, E. K. H. Ferroelectric switching in ferroelastic materials with rough surfaces. Sci. Rep. 9, 15834 (2019).

8. Aird, A. \& Salje, E. K. H. Sheet superconductivity in twin walls: experimental evidence of. J. Phys.: Condens. Matter 10, L377-L380 (1998).

9. Pellegrini, C., Glawe, H. \& Sanna, A. Density functional theory of superconductivity in doped tungsten oxides. Phys. Rev. Mater. 3, 064804 (2019).

10. Zubko, P., Catalan, G. \& Tagantsev, A. K. Flexoelectric effect in solids. Ann. Rev. Mater. Res. 43, 387-421 (2013).

11. Salje, E. K. H., Li, S., Stengel, M., Gumbsch, P. \& Ding, X. Flexoelectricity and the polarity of complex ferroelastic twin patterns. Phys. Rev. B 94, 024114 (2016).

12. Van Aert, S. et al. Direct observation of ferrielectricity at ferroelastic domain boundaries in $\mathrm{CaTiO}_{3}$ by electron microscopy. Adv. Mater. 24, 523-527 (2012).

13. Goncalves-Ferreira, L., Redfern, S. A., Artacho, E. \& Salje, E. K. Ferrielectric twin walls in $\mathrm{CaTiO}_{3}$. Phys. Rev. Lett. 101, 097602 (2008).

14. Barone, P., Di Sante, D. \& Picozzi, S. Improper origin of polar displacements at $\mathrm{CaTiO}_{3}$ and $\mathrm{CaMnO}_{3}$ twin walls. Phys. Rev. B 89, 144104 (2014).

15. Zubko, P., Gariglio, S., Gabay, M., Ghosez, P. \& Triscone, J.-M. Interface physics in complex oxide heterostructures. Annu. Rev. Condens. Matter Phys. 2, 141-165 (2011).

16. Christensen, D. V. et al. Stimulating oxide heterostructures: a review on controlling $\mathrm{SrTiO}_{3}$-based heterointerfaces with external stimuli. Adv. Mater. Interfaces 6, 1900772 (2019).

17. Pai, Y.-Y., Tylan-Tyler, A., Irvin, P. \& Levy, J. Physics of $\mathrm{SrTiO}_{3}$-based heterostructures and nanostructures: a review. Rep. Prog. Phys. 81, 036503 (2018).

18. Wang, D., Salje, E. K. H., Mi, S.-B., Jia, C.-L. \& Bellaiche, L. Multidomains made of different structural phases in multiferroic $\mathrm{BiFeO}_{3}$ : A first-principles-based study. Phys. Rev. B 88, 134107 (2013).

19. Ohtomo, A. \& Hwang, H. Y. A high-mobility electron gas at the $\mathrm{LaAlO}_{3} / \mathrm{SrTiO}_{3}$ heterointerface. Nature 427, 423-426 (2004).

20. Tra, V. T. et al. Ferroelectric control of the conduction at the $\mathrm{LaAlO}_{3} / \mathrm{SrTiO}_{3}$ heterointerface. Adv. Mater. 25, 3357-3364 (2013).

21. Reinle-Schmitt, M. L. et al. Tunable conductivity threshold at polar oxide interfaces. Nat. Commun. 3, 932 (2012).

22. Biscaras, J. et al. Two-dimensional superconducting phase in $\mathrm{LaTiO}_{3} / \mathrm{SrTiO}_{3}$ heterostructures induced by high-mobility carrier doping. Phys. Rev. Lett. 108, 247004 (2012)

23. Li, L., Richter, C., Mannhart, J. \& Ashoori, R. C. Coexistence of magnetic order and two-dimensional superconductivity at $\mathrm{LaAlO}_{3} / \mathrm{SrTiO}_{3}$ interfaces. Nat. Phys. 7, 762-766 (2011).

24. Christensen, D. V. et al. Strain-tunable magnetism at oxide domain walls. Nat. Phys. 15, 269-274 (2018).

25. Frenkel, Y., Xie, Y., Hwang, H. Y. \& Kalisky, B. Magnetism and conductivity along structural domain walls of $\mathrm{SrTiO}_{3}$. J. Supercond. Nov. Magn. 33, 195-197 (2019).

26. Juraschek, D. M. et al. Dynamical magnetic field accompanying the motion of ferroelectric domain walls. Phys. Rev. Lett. 123, 127601 (2019).

27. Juraschek, D. M., Fechner, M., Balatsky, A. V. \& Spaldin, N. A. Dynamical multiferroicity. Phys. Rev. Mater. 1, 014401 (2017).

28. Harrison, R. J. \& Salje, E. K. H. Ferroic switching, avalanches, and the Larkin length: Needle domains in $\mathrm{LaAlO}_{3}$. Appl. Phys. Lett. 99, 151915 (2011).

29. Puchberger, $\mathrm{S}$. et al. The noise of many needles: Jerky domain wall propagation in $\mathrm{PbZrO}_{3}$ and $\mathrm{LaAlO}_{3}$. APL Mater. 5, 046102 (2017).

30. Hui, Q., Tucker, M. G., Dove, M. T., Wells, S. A. \& Keen, D. A. Total scattering and reverse Monte Carlo study of the $105 \mathrm{~K}$ displacive phase transition in strontium titanate. J. Phys.: Condens. Matter 17, S111-S124 (2005).

31. Salje, E. K., Aktas, O., Carpenter, M. A., Laguta, V. V. \& Scott, J. F. Domains within domains and walls within walls: evidence for polar domains in cryogenic $\mathrm{SrTiO}_{3}$. Phys. Rev. Lett. 111, 247603 (2013).

32. Frenkel, Y. et al. Imaging and tuning polarity at $\mathrm{SrTiO}_{3}$ domain walls. Nat. Mater. 16, 1203-1208 (2017).

33. Lu, G., Li, S., Ding, X. \& Salje, E. K. H. Piezoelectricity and electrostriction in ferroelastic materials with polar twin boundaries and domain junctions. Appl. Phys. Lett. 114, 202901 (2019).

34. He, X. et al. The interaction between vacancies and twin walls, junctions, and kinks, and their mechanical properties in ferroelastic materials. Acta Mater. 178, 26-35 (2019). 
35. Li, S. et al. Strain-controlled thermal conductivity in ferroic twinned films. Sci. Rep. 4, 6375 (2014).

36. Lu, G., Li, S., Ding, X., Sun, J. \& Salje, E. K. H. Enhanced piezoelectricity in twinned ferroelastics with nanocavities. Phys. Rev. Mater. 4, 074410 (2020).

37. Salje, E. Phase transitions in ferroelastic and co-elastic crystals. Ferroelectrics 104, 111-120 (1990).

38. Scott, J. F., Salje, E. K. H. \& Carpenter, M. A. Domain wall damping and elastic softening in $\mathrm{SrTiO}_{3}$ : evidence for polar twin walls. Phys. Rev. Lett. 109, 187601 (2012).

39. Ding, X. et al. Statistics of twinning in strained ferroelastics. J. Phys.: Condens. Matter 29, 394002 (2017).

40. Salje, E. K. H., Buckley, A., van Tendeloo, G., Ishibashi, Y. \& Nord, G. L. Needle twins and right-angled twins in minerals; comparison between experiment and theory. Am. Miner. 83, 811-822 (1998).

41. Bismayer, U., Hensler, J., Salje, E. \& Güttler, B. Renormalization phenomena in Badiluted ferroelastic lead phosphate, $\left(\mathrm{Pb}_{1-\mathrm{x}} \mathrm{Ba}_{\mathrm{x}}\right)_{3}\left(\mathrm{PO}_{4}\right)_{2}$. Phase Transit. 48, 149-168 (1994).

42. Daraktchiev, M., Salje, E. K. H., Lee, W. T. \& Redfern, S. A. T. Effect of internal friction on transformation twin dynamics in perovskite $\mathrm{Sr}_{x} \mathrm{Ba}_{1-\mathrm{x}} \mathrm{SnO}_{3}(\mathrm{x}=0.6,0.8)$. Phys. Rev. B 75, 134102 (2007).

43. Harrison, R. J., Redfern, S. A. T. \& Salje, E. K. H. Dynamical excitation and anelastic relaxation of ferroelastic domain walls in $\mathrm{LaAlO}_{3}$. Phys. Rev. B 69, 144101 (2004).

44. Novak, J., Bismayer, U. \& Salje, E. K. H. Simulated equilibrium shapes of ferroelastic needle domains. J. Phys.: Condens. Matter 14, 657-664 (2002).

45. Lu, G., Li, S., Ding, X., Sun, J. \& Salje, E. K. H. Electrically driven ferroelastic domain walls, domain wall interactions, and moving needle domains. Phys. Rev. Mater. 3, 114405 (2019).

46. Salje, E. K. H., Wang, X., Ding, X. \& Scott, J. F. Ultrafast switching in avalanchedriven ferroelectrics by supersonic kink movements. Adv. Funct. Mater. 27, 1700367 (2017).

47. Jackson, J. D. Classical Electrodynamics. 5, 184-187 (Wiley, 1998).

48. Harrison, R. J., Redfern, S. A. T., Buckley, A. \& Salje, E. K. H. Application of real-time, stroboscopic $x$-ray diffraction with dynamical mechanical analysis to characterize the motion of ferroelastic domain walls. J. Appl. Phys. 95, 1706-1717 (2004).

49. Everhardt, A. S. et al. Periodicity-Doubling Cascades: Direct Observation in Ferroelastic Materials. Phys. Rev. Lett. 123, 087603 (2019).

50. Salje, E. \& Wruck, B. Specific-heat measurements and critical exponents of the ferroelastic phase transition in $\mathrm{Pb}_{3}\left(\mathrm{PO}_{4}\right)_{2}$ and $\mathrm{Pb}_{3}\left(\mathrm{P}_{1-x} \mathrm{As}_{x} \mathrm{O}_{4}\right)_{2}$. Phys. Rev. B 28, 6510 (1983).

51. Li, S., Bismayer, U., Ding, X. \& Salje, E. K. H. Ferroelastic shear bands in $\mathrm{Pb}_{3}\left(\mathrm{PO}_{4}\right)_{2}$. Appl. Phys. Lett. 108, 022901 (2016).

52. Nosé, S. A unified formulation of the constant temperature molecular dynamics methods. J. Chem. Phys. 81, 511-519 (1984).

53. Hoover, W. G. Canonical dynamics: Equilibrium phase-space distributions. Phys. Rev. A 31, 1695-1697 (1985).

54. Plimpton, S. Fast parallel algorithms for short-range molecular-dynamics. J. Comput. Phys. 117, 1-19 (1995).

\section{ACKNOWLEDGEMENTS}

X.D. and J.S. are grateful to NSFC $(51320105014,51621063)$ and the 111 project (BP 2018008) for financial support. E.K.H.S. is grateful to EPSRC (EP/P024904/1) for support. S.L. acknowledges the support from NKRDPC (2019YFA0307900).

\section{AUTHOR CONTRIBUTIONS}

E.K.H.S. conceived and designed the project. G.L. and S.L. performed molecular dynamics simulations. G.L., S.L., X.D., J.S., and E.K.H.S. analysed data. G.L., S.L., X.D., and E.K.H.S. wrote the paper. All authors contributed to discussions of the results.

\section{COMPETING INTERESTS}

The authors declare no competing interests.

\section{ADDITIONAL INFORMATION}

Supplementary information is available for this paper at https://doi.org/10.1038/ s41524-020-00412-5.

Correspondence and requests for materials should be addressed to S.L., X.D. or E. K. H.S.

Reprints and permission information is available at http://www.nature.com/ reprints

Publisher's note Springer Nature remains neutral with regard to jurisdictional claims in published maps and institutional affiliations.

cc) (i)

Open Access This article is licensed under a Creative Commons Attribution 4.0 International License, which permits use, sharing, adaptation, distribution and reproduction in any medium or format, as long as you give appropriate credit to the original author(s) and the source, provide a link to the Creative Commons license, and indicate if changes were made. The images or other third party material in this article are included in the article's Creative Commons license, unless indicated otherwise in a credit line to the material. If material is not included in the article's Creative Commons license and your intended use is not permitted by statutory regulation or exceeds the permitted use, you will need to obtain permission directly from the copyright holder. To view a copy of this license, visit http://creativecommons. org/licenses/by/4.0/.

(c) The Author(s) 2020 